\title{
Zur Syntax adverbialer Satzverknüpfungen: Deutsch - Italienisch - Portugiesisch
}

für Gisela Zifonun zum 29.03.2011

\begin{abstract}
Der vorliegende Beitrag untersucht die grammatische Realisierung satzförmiger und satzwertiger Verbgruppen- und Satzadverbialia im Deutschen im Vergleich mit den romanischen Sprachen Italienisch und Portugiesisch (schwerpunktmäßig in der brasilianischen Varietät). Solche Adverbialia können formal recht unterschiedlich realisiert werden. Für das Deutsche sind finite, subjunktor-eingeleitete adverbiale Nebensätze typisch. Seltener sind uneingeleitete finite Nebensätze, Partizipialgruppen und durch eine Präposition eingeleitete Infinitivgruppen. In den romanischen Sprachen werden Gerundial-, Partizipial- und Infinitivgruppen deutlich häufiger als Adverbialia genutzt. Anders als im Deutschen können sie auch eigene Subjekte haben, wodurch sie finiten Nebensätzen ähnlicher werden.
\end{abstract}

\section{Einleitung}

Thema dieses Beitrags ${ }^{1}$ sind Adverbialia mit verbalem Kern, genauer gesagt: satzförmige und satzwertige Verbgruppen- und Satzadverbialia. Als satzförmig und satzwertig fasse ich Ausdrücke zusammen, die aus einem Verb und seinen Ergänzungen bestehen. Satzförmig sind Ausdrücke mit einer finiten Verbform und eigenem Subjekt; satzwertig sind Ausdrücke mit einer infiniten Verbform (Partizip, Gerundium, Infinitiv), denen ein eigenes Subjekt fehlt oder fehlen kann, die aber trotzdem satztypische syntaktische und semantische Eigenschaften aufweisen (vgl. Zifonun/Hoffmann/Strecker 1997, S. 2162). Als Verbgruppen- und Satzadverbialia bezeichne ich Ausdrücke, die an eine Erweiterung eines Matrixverbs bzw. an einen Satz adjungiert sind (vgl. ebd., S. 1124 ff., 1177 ff., 2275 ff.). Auf die Unterschiede zwischen Verbgruppen- und Satzadverbialia gehe ich in diesem Aufsatz nicht ein. Insbesondere lasse ich offen, welche der untersuchten Ausdrücke zur ersten und welche zur zweiten Gruppe zu rechnen sind.

Ich danke Joachim Born, Noah Bubenhofer, Marina Foschi Albert, Lutz Gunkel, Selma M. Meireles, Adriano Murelli, Fábio Nogueira, Liudmila Olalde, Miriam Ravetto, Tinka Reichmann, Bernd Wiese, Gisela Zifonun und einem anonymen Gutachter für Ratschläge, Diskussion und Hilfe bei der Beurteilung und Übersetzung von Belegen. 
Ich behandle fünf Realisierungsmöglichkeiten für Adverbialia mit verbalem Kern, die in allen drei Vergleichssprachen bestehen, aber unterschiedlich genutzt werden (vgl. Zifonun/Hoffmann/Strecker 1997, S. 1145 ff., 1430 ff., 2205 ff., 2275 ff.; Duden 2009, S. 1048 f.; Serianni 1989, S. 573 ff.; Renzi et al. 2001, Bd. II, S. 553 ff., 571 ff., 593 ff., 720 ff.; Gärtner 1998, S. 343 ff.; GPB, Bd. III, 2009, S. 193 ff., 206 ff.):

- durch einen Subjunktor eingeleitete Nebensätze,

- uneingeleitete Nebensätze,

- uneingeleitete Partizipial- bzw. Gerundialgruppen,

- durch einen Subjunktor oder eine Präposition eingeleitete Partizipialbzw. Gerundialgruppen,

- durch eine Präposition eingeleitete Infinitivgruppen.

\section{Subjunktor-eingeleitete adverbiale Nebensätze}

\subsection{Deutsch}

Für das Deutsche können subjunktor-eingeleitete finite Nebensätze als die prototypische Realisierung von Adverbialia mit verbalem Kern gelten (vgl. Kortmann 1997, S. 238 ff.). Ich gebe je ein Beispiel für einen temporal situierenden, einen kausalen und einen konzessiven Adverbialsatz, einmal in Mittelfeldstellung, einmal vorangestellt und einmal nachgestellt:

(1) Gerüstbauteile müssen, nachdem sie mit asbesthaltigen Erzeugnissen in Kontakt gekommen sind, gereinigt werden. ${ }^{2}$

(2) Weil wir zuerst nicht alles verstanden hatten, gab uns der Lehrer noch einen Lückentext.

(3) Die Kritik an der Regierung ist nie verebbt, gleichwohl sie weniger geworden ist.

Die meisten Adverbialsätze können alle drei Stellungen einnehmen (vgl. Duden 2009, S. 1052). Lediglich Konsekutivsätze müssen generell nachgestellt werden (vgl. Zifonun/Hoffmann/Strecker 1997, S. 2307 ff.). Für

Alle Beispiele sind Originalbelege oder stammen aus der referierten Fachliteratur. Zum Teil wurden sie leicht gekürzt oder bearbeitet. Einige wenige Belege wurden einem kleinen Korpus fiktionaler Texte entnommen, das aus den Romanen „Der Vorleser“ von Bernhard Schlink (1995) und seinen Übersetzungen ins Italienische und Portugiesische, „Dois irmãos“ von Milton Hatoum (2000) und seinen Übersetzungen ins Italienische und Deutsche sowie „La solitudine dei numeri primi“" von Paolo Giordano (2008) und seinen Übersetzungen ins Deutsche und Portugiesische besteht. Die meisten Belege wurden mit der Suchmaschine Google auf deutsch-, italienisch- und portugiesischsprachigen Internetseiten in den Domänen .de, it und .br gefunden. Alle italienischen und portugiesischen Belege und ihre Übersetzungen/ Glossen wurden von Muttersprachlern überprüft. Auf den Nachweis der Fundorte wird ökonomiehalber verzichtet. 
kausale zumal-Sätze ist Nachstellung bevorzugt (vgl. Pasch et al. 2003, S. 430 f.). Die Autoren des „Handbuchs der deutschen Konnektoren“ bezeichnen Ausdrücke wie sodass und zumal deshalb als Postponierer (ebd., S. 418 ff.).

Subjunktor-eingeleitete Nebensätze haben - wenn man von subjektlosen Sätzen wie weil mir kalt ist einfachheitshalber einmal absieht - stets ein eigenes explizites Subjekt. Im Deutschen weisen sie Endstellung des Finitums auf. In der Linearstruktur, die gewöhnlich mit dem so genannten Felderschema (vgl. Höhle 1986; Sternefeld 2008, S. 286 ff.) beschrieben wird, nimmt der Subjunktor die linke Klammerposition ein. Diese ist damit für das Finitum unzugänglich. Das Finitum muss in seiner Grundstellung am rechten Rand der rechten Klammerposition verbleiben:

\begin{tabular}{|l|l|l|l|l|}
\hline VF & \multicolumn{1}{|c|}{ LK } & \multicolumn{1}{|c|}{ MF } & \multicolumn{1}{c|}{ RK } & NF \\
\hline & nachdem & sie mit Asbest in Kontakt & gekommen sind & \\
\hline & weil & wir zuerst nicht alles & verstanden hatten & \\
\hline & gleichwohl & sie weniger & geworden ist & \\
\hline
\end{tabular}

Abb. 1: Linearstruktur subjunktor-eingeleiteter Nebensätze im Deutschen (VF - Vorfeld, LK - linke Klammerposition, MF - Mittelfeld, RK - rechte Klammerposition, NF - Nachfeld)

Das Inventar der Subjunktoren ist im Deutschen, verglichen mit anderen europäischen Sprachen (vgl. Kortmann 1997, S. 245), relativ groß. Im „Handbuch der deutschen Konnektoren“" (Pasch et al. 2003, S. 354, 418) sind ca. 50 monolexematische Subjunktoren und Postponierer gelistet. Hinzu kommen ca. 40 Ausdrücke, die aus mehreren Wörtern zusammengesetzt sind und insgesamt als Einleiter finiter Adverbialsätze dienen können wie abgesehen davon, dass oder unbeschadet dessen, dass. Solche Ausdrücke können in gewissem Maße auch neu gebildet werden.

Die Subjunktoren und Postponierer des Deutschen decken ein breites Feld semantischer Relationen ab. Nur rein additive und disjunktive Verknüpfungen, für die und und oder stehen, können im Deutschen kaum durch Subjunktoren ausgedrückt werden (vgl. Duden 2009, S. 1076 f.). Es gibt semantisch unterspezifizierte Subjunktoren wie wenn oder indem, die ein breites Spektrum von Interpretationen zulassen, aber auch Subjunktoren wie weil oder obwohl, die die Interpretation weitgehend festlegen (vgl. Blühdorn 2010, S. 224 ff.).

\subsection{Italienisch und Portugiesisch}

Im Italienischen und Portugiesischen werden subjunktor-eingeleitete finite Nebensätze ebenfalls häufig verwendet, kommen aber in Texten anteilig et- 
was seltener vor als im Deutschen. Ich gebe ein Beispiel aus dem Italienischen für einen nachgestellten kausalen und eines aus dem Portugiesischen für einen vorangestellten temporal situierenden Adverbialsatz:

(4) Molte donne non fanno il secondo figlio perché i figli costano troppo.

Viele Frauen bekommen kein 2weites Kind, weil Kinder zu viel kosten. ${ }^{3}$

(5) Quando Oswaldo abriu a papelaria, pela manhã, havia 56 cadernos na prateleira.

Am Morgen, als Oswaldo den Schreibwarenladen öffnete, lagen 56 Hefte im Regal.

Auch im Italienischen und Portugiesischen sind Adverbialsätze sehr flexibel in ihrer Stellung (vgl. Renzi et al. 2001, Bd. II, S. 731 ff.; Gärtner 1998, S. 146). Es gibt aber Beschränkungen für einzelne Subjunktoren. Konsekutivsätze werden in beiden Sprachen wie im Deutschen stets nachgestellt (vgl. Serianni 1989, S. 585; Schwarze 1995, S. 445; Gärtner 1998, S. 471; Neves 2000, S. 915 f.). Bei den Kausalsätzen werden perché- bzw. porque-Sätze typischerweise nach-, siccome- bzw. como-Sätze dagegen so gut wie ausnahmslos vorangestellt (vgl. Serianni 1989, S. 576 f.; Renzi et al. 2001, Bd. II, S. 740; Ravetto/Blühdorn 2011, S. 218 ff.; Neves 2000, S. 808 ff.).

Im Italienischen und Portugiesischen ist die Satzstruktur nicht wie im Deutschen durch eine Verbalklammer, sondern durch die Abfolge SubjektVerb gekennzeichnet (vgl. Schwarze 1995, S. 370 ff.; Cunha/Cintra 2008, S. 176 ff.). Subjunktor-eingeleitete Nebensätze haben die gleiche syntaktische Struktur wie Hauptsätze. Der Subjunktor wird ihnen vorangestellt und nimmt den ganzen Satz als Ergänzung:

\begin{tabular}{|l|l|l|l|}
\hline & Subjekt & Verb & Objekt \\
\hline & i figli & costano & troppo \\
\hline perché & i figli & costano & troppo \\
\hline weil & Kinder & kosten & zu viel \\
\hline & Oswaldo & abriu & a papelaria \\
\hline quando & Oswaldo & abriu & a papelaria \\
\hline als & Oswaldo & öfnete & den Schreibwarenladen \\
\hline
\end{tabular}

Abb. 2: Linearstruktur subjunktor-eingeleiteter Nebensätze im Vergleich mit Hauptsätzen im Italienischen (oben) und Portugiesischen (unten)

Zu den italienischen und portugiesischen Belegen gebe ich Übersetzungen und - soweit erforderlich - wörtliche Glossen. Sie stehen in Kursivschrift. Die diskutierten Adverbialia sind in Belegen und Übersetzungen durch Fettdruck hervorgehoben. 
Wie im Deutschen haben subjunktor-eingeleitete Nebensätze in den romanischen Sprachen stets ein eigenes Subjekt, das allerdings, wenn die Referenz klar ist, an der Satzoberfläche nicht ausgedrückt werden muss (sog. Pro-Drop). Allein durch die Konjugationsform des finiten Verbs wird das Subjekt in vielen Fällen bereits eindeutig festgelegt.

Das Inventar der Subjunktoren ist in beiden Vergleichssprachen ähnlich umfangreich wie im Deutschen (vgl. Kortmann 1997, S. 245). Die Grammatiken des Italienischen (etwa Serianni 1989, S. 573 ff.; Schwarze 1995, S. 438 ff.; Renzi et al. 2001, Bd. II, S. 720 ff.) und Portugiesischen (etwa Gärtner 1998, S. 342 ff; Neves 2000, S. 787 ff.; Mateus et al. 2003, S. 705 ff.; Cunha/Cintra 2008, S. 600 ff.) führen in der Regel zwischen 50 und 100 unterordnende Konjunktionen auf. In manchen Grammatiken des Portugiesischen (z.B. bei Bechara 1999, S. 471 ff.; Mateus et al. 2003, S. 704 f.; GPB Bd. III, 2009, S. 213 ff.) werden Subjunktoren als besondere Verwendungsweisen von Präpositionen behandelt. Das wird damit gerechtfertigt, dass viele Subjunktoren morphologisch aus einer Präposition und dem Komplementierer que ('dass') ${ }^{4}$ zusammengesetzt sind, die orthographisch getrennte Wörter bilden, z.B. depois que ('nachdem'), para que ('damit'), apesar de que ('obwohl') usw. (vgl. Mioto/Kato 2002, S. 394 ff.). Die Subjunktoren des Italienischen sind großenteils genauso gebildet, werden aber überwiegend zusammengeschrieben, z.B. finché ('bis'), perché ('weil'), dacché ('seitdem', 'da') usw. Sie sind in der Grammatikschreibung fest als Konjunktionen etabliert.

Das Spektrum semantischer Relationen, das mit subjunktor-eingeleiteten Nebensätzen abgedeckt werden kann, ist in den romanischen Sprachen im Wesentlichen das gleiche wie im Deutschen. Im Italienischen und Portugiesischen kann auch die additive Verknüpfungsrelation durch einen Subjunktor (oltre che/além de que; 'zusätzlich dazu, dass') ausgedrückt werden (vgl. Gärtner 2003, S. 93 f.).

\section{Uneingeleitete adverbiale Nebensätze}

\subsection{Deutsch}

Finite adverbiale Nebensätze kommen im Deutschen - vor allem in Schrifttexten - auch uneingeleitet vor, meist als Konditionalsätze wie in (6), aber auch in adversativer (7), konzessiver (8) und kausaler (9) Verwendung (vgl. Zifonun/Hoffmann/Strecker 1997, S. 2281 f., 2298 f., 2313, 2321 f., 2324 f.; Reis/Wöllstein 2010, S. 118 ff.; König in diesem Band, Abschn. 2.2). Sie können im Vorfeld wie in (6), im Vorvorfeld wie in (7) (vgl. Zifonun/Hoffmann/ Strecker 1997, S. 1577 ff., 2289), im Mittelfeld wie in (8) und im Nachfeld bzw. Nachnachfeld des Matrixsatzes wie in (9) stehen: ${ }^{5}$

Bedeutungsparaphrasen schließe ich in hochgestellte einfache Anführungsstriche ein.

Axel/Wöllstein (2009) und Reis/Wöllstein (2010) vertreten die Ansicht, Verberst-Nebensätze seien im Gegenwartsdeutschen syntaktisch generell nicht in den Matrixsatz integriert und 
(6) Hat er einen Job, muss er die Aufnahme einer Nebentätigkeit anzeigen.

(7) Ist die Aussagenlogik noch leicht zugänglich, so bietet die Prädikatenlogik schon einige Fallstricke. (aus Reis/Wöllstein 2010, S. 123)

(8) Dennoch hätte sie, wäre sie auch noch so energisch und kompromisslos, keine Chance gegen die Obrigkeit.

(9) Er bemühte sich persönlich um die Bauplanung, wollte er doch ein Institut für die nächsten Jahrzehnte errichten.

Uneingeleitete Nebensätze haben stets ein eigenes explizites Subjekt und weisen durchweg Erststellung des Finitums auf. Das Finitum nimmt die linke Klammerposition ein, also die gleiche Position, die in einem eingeleiteten Nebensatz der Subjunktor innehat:

\begin{tabular}{|l|l|l|l|l|}
\hline VF & \multicolumn{1}{|c|}{ LK } & \multicolumn{1}{|c|}{ MF } & \multicolumn{1}{|c|}{ RK } & \multicolumn{1}{|c|}{ NF } \\
\hline & hat & er einen Job & hat & \\
\hline & wenn & er einen Job & die Aussagenlogik noch zugänglich & \\
\hline & ist & die Aussagenlogik noch zugänglich & ist & \\
\hline & während & sie auch noch so energisch & & \\
\hline & wäre & sie auch noch so energisch & wäre & \\
\hline & wenn & er doch ein Institut errichten & & \\
\hline & wollte & er doch ein Institut errichten & wollte & \\
\hline
\end{tabular}

Abb. 3: Linearstruktur uneingeleiteter und eingeleiteter adverbialer Nebensätze im Deutschen

Uneingeleitete Konditionalsätze kommen, ebenso wie eingeleitete Konditionalsätze, auch als Ergänzungen der Vergleichspartikel als vor:

(10) Ich habe oft Blasen auf der Zunge, als

(a) hätte ich mich verbrannt.

(b) wenn/ob ich mich verbrannt hätte.

Die Beobachtung, dass Verberstsätze und subjunktor-eingeleitete Verbletztsätze in solchen Umgebungen alternieren, ist eine der Evidenzen, die dafür sprechen, dass Subjunktor und vorangestelltes Finitum die gleiche Strukturposition einnehmen (vgl. Sternefeld 2008, S. 322).

könnten deshalb nicht im Vor- oder Mittelfeld stehen. Die Argumente, die sie anführen, scheinen mir aber für eine so weitgehende Schlussfolgerung nicht hinreichend. Ich bleibe hier bei der traditionellen Annahme, dass uneingeleitete Adverbialsätze in Beispielen wie (6) und (8) in den Matrixsatz integriert sind (vgl. Zifonun/Hoffmann/Strecker 1997, S. 2313 u.ö.). 


\subsection{Portugiesisch und Italienisch}

Im Portugiesischen sind uneingeleitete Nebensätze ebenfalls gebräuchlich, allerdings wohl nur als Konditionalsätze (orações subordinadas adverbiais condicionais construidas sem conjunção; Cunha/Cintra 2008, S. 179). Beispiel (11) stammt aus Neves (2000, S. 857):

(11) Fosse a Petrobras uma empresa privada, não estaria sujeita às ingerências dos políticos.

Wäre Petrobras ein Privatunternebmen, wäre es nicht den Einmischungen der Politiker ausgesetzt.

Neves (ebd., S. 858) zufolge sind uneingeleitete Konditionalsätze im Portugiesischen durchweg kontrafaktisch (angezeigt durch den Subjunktiv). Im Vergleich mit dem Deutschen bedeutet das eine stärkere funktionale Festlegung. Im Deutschen können uneingeleitete Nebensätze vielseitiger verwendet werden (vgl. Beispiele (6) bis (9)), als Konditionalsätze nicht nur kontrafaktisch (Konjunktiv), sondern auch hypothetisch (Indikativ).

Wie im Deutschen steht auch im Portugiesischen in uneingeleiteten Nebensätzen das Finitum am Satzanfang. Die Grammatiken sprechen in solchen Fällen von Subjekt-Verb-Inversion (vgl. Cunha/Cintra 2008, S. 177 ff.). Die Voranstellung des Finitums muss hier aber anders als im Deutschen erklärt werden, denn auch Sätze mit Subjekt-Verb-Inversion können noch mit dem Subjunktor se ('wenn', 'ob') eingeleitet werden:

(12) Tenho uma cachorrinha Poodle que tem 8 anos. Esses 8 anos dessa cadela seriam equivalentes a 56 anos se fosse ela uma mulher.

Ich habe eine Pudelbündin, die 8 Jahre alt ist. Die 8 Jabre dieser Hündin entsprächen 56 Jahren, wörtl.: wenn wäre sie eine Fran (wenn sie eine Fran wäre).

Im Deutschen sind Subjunktoren nicht mit vorangestellten Finita kombinierbar. Ich kann hier nicht im Detail diskutieren, wie die syntaktische Struktur von Sätzen wie (12) darzustellen ist. Dazu verweise ich auf die Grammatik von Mateus et al. (2003, S. 445 ff.; 471 ff.; vgl. auch: Müller/Riemer 1998, S. 182 ff.).

Wie im Deutschen können eingeleitete und uneingeleitete Konditionalsätze auch im Portugiesischen als Ergänzungen zu Vergleichspartikeln fungieren:

(13) Denúncias são tornadas públicas de forma leviana contra o Sumo Pontífice, como (se) fosse ele o responsável por tais atos vergonhosos.

Anschuldigungen gegen den Papst werden leichtfertig öffentlich gemacht, wörtl.: wie (wenn) wäre er der Verantwortliche (als wäre er der Verantwortliche) für solche schändlichen Taten. 
In den Grammatiken des Italienischen finden sich ebenfalls Abschnitte über uneingeleitete Konditionalsätze (vgl. Serianni 1989, S. 547, 589; Renzi et al. 2001, Bd. II, S. 771). Diese scheinen aber wesentlich weniger gebräuchlich zu sein als im Deutschen und Portugiesischen. Mehrere italienische Informanten erklärten mir kategorisch, uneingeleitete Nebensätze gebe es in ihrer Sprache nicht. Erst als ich ihnen Beispiele wie (14) vorlegte, mussten sie einräumen, dass sie in bestimmten Fällen doch vorkommen:

(14) Fossi in te, non lo farei.

Wäre ich an deiner Stelle, täte ich das nicht.

Als Ergänzungen einer Vergleichspartikel scheinen sie häufiger zu sein:

(15) La possibilità di far girare in rete contenuti facilmente prodotti attraverso cellulari o telecamere rende Internet e le nuove tecnologie ancora più interessanti agli occhi dei ragazzi. Sembra presente nei ragazzi il bisogno che i loro gesti acquisiscano notorietà, come (se) fosse questo l'unico modo per renderli consistenti.

Die Möglichkeit, im Netz Inbalte kursieren zu lassen, die mit Handys oder Webcams schnell produziert werden können, macht das Internet und die neuen Technologien für die Jugendlichen noch interessanter. Bei den Jugendlichen scheint das Bedürfnis zu bestehen, ibre Handlungen öffentlich bekannt zu machen, als wäre das die einzige Möglichkeit, ihnen Gültigkeit zu verleiben.

In Sätzen wie (14) und (15) kann der Subjunktor se ('wenn', 'ob') fakultativ stehen oder weggelassen werden. Die syntaktische Struktur sollte demnach ebenso aussehen wie die entsprechender Sätze des Portugiesischen.

\section{Uneingeleitete Partizipial- bzw. Gerundialgruppen}

\subsection{Deutsch}

Im Deutschen können Partizipialgruppen sowohl mit Präsens- als auch mit Perfektpartizip adverbial verwendet werden (vgl. Welker 1992, S. 62 ff.; Zifonun/Hoffmann/Strecker 1997, S. 2214 ff.; Duden 2009, S. 854 f.). Das gilt zum einen für Partizipien wie angenommen, vorausgesetžt u.a. (vgl. Pasch et al. 2003, S. 354 ff.), die sich grammatisch zu subjunktorähnlichen Ausdrücken, also zu Einleitern adverbialer Nebensätze weiterentwickelt haben. ${ }^{6}$ Es gilt aber auch in einem allgemeineren Sinne für frei bildbare Partizipialgruppen. In (16) sehen wir ein erweitertes Partizip Präsens im Nachfeld, in (17) und (18) erweiterte Perfektpartizipien im Vorfeld bzw. im Mittelfeld:

Auch wäbrend, gegenwartssprachlich eindeutig ein Subjunktor, ist der Form nach noch als Partizip zu erkennen. 
(16) Hegel, Hölderlin und Schelling pflanzen in Tübingen einen Freiheitsbaum und umtanzen ihn, revolutionäre Lieder singend.

(17) Angekommen in Las Vegas, stand für die Miss-Universe-Kandidatinnen ein volles Programm an.

(18) Auf dem Bild durchschreitet eine junge Frau, das Kleid geschürzt, einen Fluss.

Die Linearstruktur deutscher Partizipialgruppen kann wie die finiter Sätze mit dem Felderschema dargestellt werden. Das Partizip nimmt dabei stets die rechte Klammerposition ein. Linke Klammerposition und Vorfeld bleiben unbesetzt:

\begin{tabular}{|l|l|l|l|l|}
\hline VF & LK & \multicolumn{1}{|c|}{ MF } & \multicolumn{1}{c|}{ RK } & \multicolumn{1}{c|}{ NF } \\
\hline & & revolutionäre Lieder & singend & \\
\hline & & & angekommen & in Las Vegas \\
\hline & & das Kleid & geschürzt & \\
\hline
\end{tabular}

Abb. 4: Linearstruktur von Partizipialgruppen im Deutschen

Laut Duden-Grammatik (2009, S. 1049) werden satzwertige Partizipialgruppen ,traditionell zu den Adverbialnebensätzen gezählt“; genau genommen handele es sich aber „um prädikative Angaben (freie Prädikative). "Tatsächlich ist die Lage nicht immer so eindeutig (vgl. Zifonun/Hoffmann/Strecker 1997, S. 2216 ff.).

In vielen Fällen kann der Beitrag, den die Partizipialgruppe zur Satzbedeutung leistet, durch ein satzförmiges oder nicht-satzförmiges Attribut, nicht aber durch einen subjunktor-eingeleiteten Adverbialsatz paraphrasiert werden, so z.B. in (18):

(18) Auf dem Bild durchschreitet eine junge Frau,

(a) die das Kleid geschürzt hat, einen Fluss.

(b) mit geschürztem Kleid einen Fluss.

(c) =?? indem/wobei das/ihr Kleid geschürzt ist, einen Fluss. ${ }^{7}$

Die Partizipialgruppe könnte ohne Veränderung der deskriptiven Satzbedeutung allein ins Vorfeld bewegt werden. Demnach kann sie, zumindest optional, als Satzglied analysiert werden:

(18d) Das Kleid geschürzt, durchschreitet auf dem Bild eine junge Frau einen Fluss.

Vorangestelltes Gleichheitszeichen steht für Bedeutungsäquivalenz, durchgestrichen $(\neq)$ für Nicht-Äquivalenz, mit einem oder mehreren Fragezeichen (=? oder =??) für zweifelhafte Äquivalenz. 
Partizipialgruppen, die sich so verhalten, sind syntaktisch als lockere Attribute oder freie Prädikative zu deuten.

In anderen Fällen kann das Gemeinte nicht mit einem Relativsatz, sondern ausschließlich mit einem Adverbialsatz wiedergeben werden wie in (17). Dann ist die Partizipialgruppe als Adverbiale zu deuten:

(17a) $\neq$ Für die Miss-Universe-Kandidatinnen, die in Las Vegas angekommen waren, stand ein volles Programm an.

(17b) Als sie in Las Vegas angekommen waren, stand für die MissUniverse-Kandidatinnen ein volles Programm an.

Die syntaktische Deutung einer Partizipialgruppe wird auch durch ihre Linearstellung im Satz beeinflusst. Steht sie im Mittelfeld, so kann sie einer Nominalgruppe, insbesondere dem Subjekt des Matrixsatzes, unmittelbar folgen, wie in (18). In solchen Fällen liegt eine Deutung als Prädikativ oder Attribut stets nahe. Steht sie dagegen im Vor- oder Nachfeld des Matrixsatzes wie in (16) und (17) und/oder grenzt sie nicht unmittelbar an eine Nominalgruppe an, in die sie inkorporierbar wäre, so werden Deutungen als Adverbiale wahrscheinlicher.

Im Deutschen haben Partizipialgruppen gewöhnlich kein eigenes explizites Subjekt (vgl. Zifonun/Hoffmann/Strecker 1997, S. 2162; Duden 2009, S. 855). Das semantische Subjekt des Partizips muss aus dem Kontext erschlossen werden. Meist bietet sich dafür das Subjekt des Matrixsatzes an, wie in (16) und (18). Dass es auch andere Möglichkeiten gibt, zeigt (17), wo ein volles Programm Subjekt des Matrixsatzes, aber die Miss-Universe-Kandidatinnen semantisches Subjekt des Partizips ist. Eine detaillierte Diskussion der Faktoren, die die Erschließung des semantischen Subjekts infiniter Verben beeinflussen, findet sich in Zifonun/Hoffmann/Strecker (1997, S. $1375 \mathrm{ff}$. und $2171 \mathrm{ff}$.$) .$

Für eine syntaktische Deutung als Prädikativ kommen nur Partizipialgruppen ohne eigenes explizites Subjekt in Frage. Partizipialgruppen mit eigenem Subjekt können auf keinen Fall als Prädikative gedeutet werden. Solche Partizipialgruppen sind in den romanischen Vergleichssprachen nichts Ungewöhnliches, aber auch im Deutschen kommen sie vereinzelt vor. Ein Originalbeleg:

(19) Hinter den Reihen des Sanitätsverbandes sitzt eine Soldatin auf einem großen Stein, ihre Hände auf die Knie gestützt, der Oberkörper nach vorne gebeugt, der Kopf gesenkt.

Hier sind der Oberkörper und der Kopf offenbar als Subjekte von gebengt bzw. gesenkt zu lesen, nicht als Objekte, denn sie stehen im Nominativ, nicht im Akkusativ (vgl. die Ausführungen zum ,absoluten Akkusativ" in Zifonun/ Hoffmann/Strecker 1997, S. 2224 ff. und Duden 2009, S. 895 f.; dazu auch 
Fabricius-Hansen/Ramm in diesem Band, Abschn. 4.2). Relativsätze sind nicht geeignet, um solche Partizipialgruppen zu paraphrasieren. Adverbialsätze können eventuell in Frage kommen:

(19a) =? Hinter den Reihen des Sanitätsverbandes sitzt eine Soldatin auf einem großen Stein, ihre Hände auf die Knie gestützt, während ihr Oberkörper nach vorne gebeugt, ihr Kopf gesenkt ist.

Am besten lässt sich das Gemeinte in solchen Fällen aber wohl durch selbständige Hauptsätze wiedergeben:

(19b) Hinter den Reihen des Sanitätsverbandes sitzt eine Soldatin auf einem großen Stein, ihre Hände auf die Knie gestützt. Ihr Oberkörper ist nach vorne gebeugt, ihr Kopf ist gesenkt.

Das deutet darauf hin, dass Partizipialgruppen mit eigenem Subjekt im Deutschen auch nicht bevorzugt als Adverbialia, sondern als selbständige Ausdrücke zu deuten sind, die dem Matrixsatz nur locker an- bzw. eingefügt wurden, etwa wie weiterführende Relativsätze (vgl. Holler 2007).

\subsection{Italienisch und Portugiesisch}

Auch in den romanischen Vergleichssprachen gibt es Partizipien wie visto ('gesehen'), dato/dado ('gegeben') u.a., die sich zu lexikalisierten Nebensatzeinleitern entwickelt haben (vgl. Renzi et al. 2001, Bd. II, S. 596; Bechara 1999, S. 473, 524). Daneben werden deutlich häufiger als im Deutschen frei bildbare Partizipialgruppen als Adverbialia verwendet (vgl. Renzi et al. 2001, Bd. II, S. 593 ff.; Hundertmark-Santos Martins 1998, S. 186). Ein Beispiel aus dem Portugiesischen:

(20) O irmão se equilibrava no meio da árvore, escondido na folhagem.

Der Bruder hielt mitten im Baum das Gleichgewicht, versteckt zwischen den Blättern.

Die Partizipialgruppe in (20) enthält kein eigenes Subjekt. Semantisches Subjekt des Partizips ist das Subjekt des Matrixsatzes, o irmão ('der Bruder'). Die Partizipialgruppe kann als Prädikativ zu o irmão oder als Adverbiale gelesen werden. Im ersten Fall kann sie durch einen Relativsatz, im zweiten durch einen finiten Adverbialsatz, z.B. mit enquanto ('während'), paraphrasiert werden:

(20a) O irmão, que estava escondido na folhagem, se equilibrava no meio da árvore.

Der Bruder, der zwischen den Blättern versteckt war, hielt mitten im Baum das Gleichgewicht. 
(20b) O irmão se equilibrava no meio da árvore, enquanto estava escondido na folhagem.

Der Bruder hielt mitten im Baum das Gleichgewicht, während/wobei er zwischen den Blättern versteckt war.

Ein Beispiel aus dem Italienischen, bei dem eine adverbiale Deutung der Partizipialgruppe präferiert ist:

(21) Giunti a destinazione il capitano e i suoi uomini dovranno fronteggiare una forma di vita aliena.

Am Ziel angekommen, müssen der Kapitän und seine Männer sich mit außerirdischen Lebewesen auseinandersetzen.

In beiden Sprachen sind auch Partizipialgruppen mit eigenem explizitem Subjekt frequent, und zwar bei Partizipien so genannter inakkusativer Verben und in inakkusativen Konstruktionen (vgl. Serianni 1989, S. 609; Renzi et al. 2001, Bd. I, S. 55 ff., 595 ff.; Mateus et al. 2003, S. 300 f., 509 ff.; Cunha/ Cintra 2008, S. 509 f.). Grob gesagt handelt es sich dabei um intransitive Verben mit nicht-agentivischem Subjekt und um Passivsätze. Im Italienischen werden alle Verben und Verbformen dazugerechnet, die das Perfekt mit essere ('sein') bilden. Ein Beispiel aus dem Italienischen, eines aus dem Portugiesischen:

(22) Arrivato il rimborso gli ho dato feedback positivo. wörtl.: Eingegangen die Erstattung (als die Erstattung eingegangen war), gab ich ihm Bescheid.

(23) Chegado o momento, tirou-se o pano que velava o quadro. wörtl.: Gekommen der Moment (als der Moment gekommen war), nahm man das Tuch ab, mit dem das Bild verbüllt war.

Subjekthaltige Partizipialgruppen können nicht als Prädikative, sondern nur als Adverbialia gelesen werden. Übersetzt man sie ins Deutsche, so muss man sie durch subjunktor-eingeleitete Nebensätze wiedergeben.

Auch Partizipialgruppen mit transitiven Verben können subjekthaltig sein, wenn sie passivisch gedeutet werden (vgl. Serianni 1989, S. 482; Schwarze 1995, S. 212 f.; Mateus et al. 2003, S. 727 f.). Ein Beispiel aus dem Italienischen, zwei aus dem Portugiesischen:

(24) Ascoltato il testimone, il pm Letizia Ruggeri ha deciso di archiviare il caso.

wörtl.: Angehört der Zeuge (nachdem der Zeuge angehört worden war), entschied die Staatsanwältin Letizia Ruggeri, das Verfahren einzustellen.

(25) Dito tudo isso só nos resta agir.

wörtl.: Gesagt dies alles (da dies alles gesagt ist), bleibt uns nur zu bandeln. 
(26) Uma vez a casa construída, é urgente que ela seja cuidada por uma doméstica.

wörtl.: Einmal das Haus gebaut (wenn das Haus fertig ist), muss man unbedingt dafür sorgen, dass sich eine Hausangestellte darum kümmert.

Dass die Nominalgruppen, die auf das Partizip folgen, in solchen Sätzen Subjektstatus haben, erkennt man an der Kongruenz. Ascoltat-o (mask. sg.) in (24) stimmt in Genus und Numerus mit il testimone überein; ascoltato il testimone wäre zu essendo stato ascoltato il testimone (wörtl.: 'seiend worden angehört der Zeuge') zu expandieren. Ebenso kongruiert dit-o (mask. sg.) in (25) mit tudo isso; dito tudo isso kann zu tendo sido dito tudo isso (wörtl.: 'seiend worden gesagt all dies') erweitert werden. ${ }^{8}$ Construid-a (fem. sg.) in (26) kongruiert mit a casa; a casa construida kann zu tendo sido a casa construida (wörtl.: 'seiend worden das Haus gebaut') erweitert werden.

Im Italienischen wie im Portugiesischen steht das Subjekt in subjekthaltigen Partizipialgruppen bevorzugt postverbal wie in (24) und (25). Im Portugiesischen ist nach uma vez ('einmal', 'wenn', 'als') auch präverbale Subjektstellung wie in (26) möglich (vgl. Mateus et al. 2003, S. 727 f.). Das Gleiche gilt im Italienischen nach una volta (vgl. Renzi et al. 2001, Bd. II, S. 599 f.):

(27) Una volta sua sorella entrata in convento, Carlo poteva disporre del patrimonio come voleva. (aus Renzi et al. 2001, Bd. II, S. 599) wörtl.: Einmal seine Schwester eingetreten ins Kloster (als seine Schwester ins Kloster gegangen war), konnte Carlo frei über den Besitzvverfügen.

Abbildung 5 gibt einen Überblick über die Stellungsmöglichkeiten:

\begin{tabular}{|l|l|l|l|l|}
\hline & Subjekt & Verb & Subjekt & \\
\hline & & giunti & & a destinazione \\
\hline & & arrivato & il rimborso & \\
\hline una volta & sua sorella & entrata & & in convento \\
\hline & & escondido & & na folhagem \\
\hline uma vez & a casa & chegado & o momento & \\
\hline
\end{tabular}

Abb. 5: Linearstruktur von Partizipialgruppen im Italienischen (oben) und Portugiesischen (unten)

$8 \quad$ Es ist aufschlussreich, Satz (25) mit (42) aus dem Deutschen (siehe unten Abschnitt 5.3) zu vergleichen. Im portugiesischen Beispiel ist tudo isso Passiv-Subjekt. Im deutschen Beispiel ist dies (ersetzbar etwa durch diesen Satz) eher als Aktiv-Objekt zu deuten. 
Der syntaktische Status von una volta/ uma vez in Sätzen wie (26) und (27) ist unklar. Ich betrachte sie hier als vorangestellte Adverbialia. Stuft man sie als Subjunktoren ein, so wären die Beispiele als eingeleitete Partizipialgruppen (siehe nächster Abschnitt) zu behandeln. Dann wäre zu schlussfolgern, dass das Subjekt in uneingeleiteten Partizipialgruppen immer postverbal stehen muss (vgl. Schwarze 1995, S. 212).

Serianni (1989, S. 482) erwähnt Fälle, in denen das Partizip eines transitiven Verbs in Genus und Numerus nicht mit der nachfolgenden Nominalgruppe kongruiert:

(28) Il signore entrò, e girato un'occhiata per la stanza, vide Lucia ravvolta nel suo cantuccio e quieta. (Manzoni, I Promessi Sposi, 1827)

Der Herr trat ein, und, als er sich im Zimmer umgeschaut hatte, sah er Lucia still in ibrer Ecke kauern.

Solche Beispiele geben seiner Darstellung nach aber einen Sprachgebrauch wieder, der heute nicht mehr aktuell ist. Ob man annehmen soll, dass ihnen ein Gerundium mit dem Hilfsverb avere ('haben') zugrundeliegt (avendo girato un'occhiata; 'habend sich umgeschaut'), sodass die nachfolgende Nominalgruppe (un'occhiata) nicht als Passiv-Subjekt, sondern als Aktiv-Objekt zu deuten wäre, ist Serianni zufolge umstritten.

In den Beispielen (20) bis (28) liegen Perfektpartizipien vor. Präsenspartizipien wie italienisch amante ('liebend', 'Liebhaber') oder tremante ('zitternd'), portugiesisch corrente ('laufend', 'Kette') oder radiante ('strahlend') werden gegenwartssprachlich meist adjektivisch oder substantivisch, kaum adverbial verwendet (vgl. Serianni 1989, S. 483; Renzi et al. 2001, Bd. II, S. 604 ff.; Bechara 1999, S. 224; Endruschat 2004, S. 270 ff.). Adverbial wird stattdessen das Gerundium - italienisch: amando ('liebend'), tremando ('zitternd'), portugiesisch: correndo ('laufend'), radiando ('strahlend') - gebraucht. Je ein Beispiel:

(29) Come tutti sappiamo gli insegnanti sono dei fannulloni che rubano lo stipendio, lavorando solo diciotto ore a settimana e con due mesi di ferie.

Wie wir alle wissen, sind die Lehrer Nichtstuer, die ibr Gehalt steblen, wörtl.: arbeitend nur achtzebn Stunden pro Woche und mit zwei Monaten Ferien (indem sie nur achtzehn Stunden pro Woche arbeiten und dann auch noch zwei Monate Ferien machen).

(30) Rosalba toma posse criticando o antecessor.

Rosalba übernimmt das Amt, wörtl.: kritisierend den Vorgänger (wobei er seinen Vorgänger kritisiert).

In beiden Beispielen ist das Subjekt des Matrixsatzes (gli insegnanti bzw. Rosalba) zugleich als semantisches Subjekt der Gerundialgruppe zu lesen. Gerundialgruppen können in beiden Sprachen aber auch relativ frei mit 
eigenem Subjekt auftreten (vgl. Serianni 1989, S. 485, 580 ff.; Renzi et al. 2001, Bd. II, S. 572 f.; Simões 2009, S. 420 ff.). Hier besteht keine Beschränkung auf inakkusative oder passivische Verben. Je zwei Beispiele:

(31) Sulle note di Rossini, Verdi e Puccini, il direttore d'orchestra, Kristjan Järvi, è stato straordinario. Il culmine di questa serata è stato con 'Rhapsody in Blue' di Gershwin, il direttore essendo accompagnato da Stefano Bollani, un pianista di grande valore.

Zu den Noten von Rossini, Verdi und Puccini war der Dirigent Kristjan Järvi außerordentlich. Der Höhepunkt des Abends war „Rhapsody in Blue" von Gershwin, wörtl.: der Dirigent seiend begleitet von Stefano Bollani, einem ausgezeichneten Pianisten (wobei dem Dirigenten mit Stefano Bollani ein ausgezeichneter Pianist zur Seite stand).

(32) Avendo dato il direttore il suo consenso, abbiamo acquistato i nuovi PC.

wörtl.: Habend gegeben der Direktor seine Zustimmung (nachdem der Direktor zugestimmt hatte), schafften wir die neues PCs an.

(33) Andou ao meu lado, uma das mãos segurando a minha pasta e a outra sobre o meu braço.

Sie ging neben mir, wörtl.: eine der Hände baltend meine Tasche (in der einen Hand meine Tasche) und die andere auf meinem Arm.

(34) Sendo você o líder, seu cão logo abandonará este hábito.

wörtl.: Seiend $d u$ der Chef (wenn du der Chef bist), wird dein Hund diese Angewohnheit schnell aufgeben.

In Gerundialgruppen beider Sprachen kann das Subjekt präverbal wie in (31) und (33) oder postverbal wie in (32) und (34) stehen (vgl. Renzi et al. 2001, Bd. II, S. 584; Simões 2009, S. 420):

\begin{tabular}{|l|l|l|l|l|}
\hline & Subjekt & Verb & Subjekt & \\
\hline & & lavorando & & diciotto ore a settimana \\
\hline & il direttore & $\begin{array}{l}\text { essendo } \\
\text { accompagnato }\end{array}$ & & da Stefano Bollani \\
\hline & & avendo dato & il direttore & il suo consenso \\
\hline & criticando & & o antecessor \\
\hline & uma das mãos & segurando & & a minha pasta \\
\hline & & sendo & você & o líder \\
\hline
\end{tabular}

Abb. 6: Linearstruktur von Gerundialgruppen im Italienischen (oben) und Portugiesischen (unten) 
Im Italienischen ist postverbale Stellung des Subjekts der Normalfall, aber nicht ausnahmslos, wie manchmal behauptet wird (etwa bei Serianni 1989, S. 580 und Schwarze 1995, S. 219). Nach Renzi et al. (2001, Bd. II, S. 573) kam präverbale Subjektstellung in älterem Italienisch häufiger vor als in der Gegenwartssprache. Im heutigen Italienisch ist sie kennzeichnend für weiterführende Gerundialgruppen, die nur locker an den Matrixsatz angeknüpft sind (vgl. ebd., S. 588 ff.).

Semantisch sind adverbiale Partizipial- und Gerundialgruppen in den romanischen Sprachen stark unterbestimmt. Sie lassen weitgehend offen, wie die semantische Beziehung zwischen Matrixsatz und Adverbiale zu deuten ist (vgl. Serianni 1989, S. 485, 580 ff.; Schwarze 1995, S. 217 f.; Hundertmark-Santos Martins 1998, S. 181 ff.; Bechara 1999, S. 523 ff.). Sie können im Sinne einer Art und Weise, aber auch temporal, konditional, kausal, konsekutiv oder konzessiv gelesen werden (vgl. Renzi et al. 2001, Bd. II, S. 576 ff., 600 ff.; Braga 1996, S. 231 ff.; Simões 2009, S. 452 ff.). Der Interpret muss im Kontext entscheiden, was für eine Deutung vermutlich den Mitteilungsabsichten des Sprechers am nächsten kommt. Diese Beobachtung hat zu der sprachkritischen Forderung geführt, in Rechtstexten, in denen es auf semantische Genauigkeit ankommt, Partizipial- und Gerundialkonstruktionen zu meiden und stattdessen subjunktor-eingeleitete Nebensätze zu verwenden (vgl. Soffritti 2010; Endruschat 2004, S. 280).

\section{Durch Subjunktoren oder Präpositionen eingeleitete Partizipial- bzw. Gerundialgruppen}

\subsection{Portugiesisch}

Im Portugiesischen müssen adverbiale Partizipial- und Gerundialgruppen allerdings nicht unbedingt semantisch unterbestimmt bleiben (vgl. Gärtner 2003, S. 92 ff.). Vor allem in informellen Texten und in der gesprochenen Sprache des Brasilianischen werden sie manchmal durch Subjunktoren oder Präpositionen eingeleitet (vgl. Hundertmark-Santos Martins 1998, S. 181; Mateus et al. 2003, S. 726 f.). Auf diese Weise können die adverbialen Relationen genau wie bei finiten, subjunktor-eingeleiteten Adverbialsätzen präzisiert werden. Zunächst ein Beispiel für eine vorangestellte subjunktoreingeleitete Gerundialgruppe ohne eigenes Subjekt:

(35) Quando trabalhando em grupo, os alunos realmente se concentram no tema.

wörtl.: Wenn arbeitend in Gruppe (wenn sie in Gruppen arbeiten), konzentrieren sich die Schüler wirklich auf das Thema.

Solche Gerundialgruppen sind finiten Adverbialsätzen syntaktisch und semantisch sehr ähnlich und können im Deutschen kaum anders als durch finite Adverbialsätze wiedergegeben werden. 
Gerundialgruppen mit eigenem explizitem Subjekt können ebenfalls durch einen Subjunktor eingeleitet werden. Dieser Konstruktionstyp ist relativ selten und nicht gut erforscht. Soweit ich sehen kann, ist er bei inakkusativen Verben und Konstruktionen eher zu erwarten als bei sonstigen Verben. Das Subjekt muss dann postverbal stehen. Ein Beispiel aus einer Politikerbiographie:

(36) Vamos encontrá-lo disposto a voltar para a chefia do governo municipal, depois de 1945, quando surgindo a normalidade democrática.

Wir werden ibn bereit sehen, an die Spitze der Gemeinderegierung zurückzukehren, nach 1945, wörtl.: als aufkommend die Normalität demokratische (als die Demokratie zur Normalität wird).

Unter den Subjunktoren scheinen sich bei Gerundialgruppen bevorzugt diejenigen zu finden, die mit zeitlicher Überlappung der beschriebenen Sachverhalte kompatibel sind, etwa quando ('wenn', 'als'), enquanto ('während') oder embora ('obwohl').

Auch Partizipialgruppen können im Portugiesischen durch Subjunktoren bzw. Präpositionen eingeleitet werden, insbesondere durch solche, die mit einer zeitlichen Abfolge der beschriebenen Sachverhalte verträglich sind, wie depois de ('nach'), antes de ('(be)vor') oder porque ('weil'). Ein Beispiel ohne eigenes Subjekt:

(37) Divinaldo foi surpreendido com 6 tiros e depois de caído ainda foi brutalmente atingido a golpes de facão.

Divinaldo wurde mit sechs Schüssen empfangen und, wörtl.: nach gefallen (als er am Boden lag), noch von brutalen Scblägen mit dem Buschmesser getroffen.

Es kommen auch subjunktor- bzw. präpositions-eingeleitete Partizipialgruppen mit eigenem Subjekt vor - wiederum ein relativ seltener und meiner Kenntnis nach kaum erforschter Konstruktionstyp:

(38) Fantasmas aparecem em quase todas as culturas, bem antes de surgido o espiritismo.

Geister erscheinen in fast allen Kulturen, wörtl:: gut vor aufgekommen der Spiritismus (weit vor dem Aufkommen des Spiritismus).

\subsection{Italienisch}

Im Italienischen werden eingeleitete Gerundial- und Partizipialgruppen demgegenüber offenbar nur selten verwendet. Belege für eingeleitete Gerundialgruppen konnte ich nur mit Konzessiv-Subjunktoren wie sebbene ('obwohl') finden (vgl. Serianni 1989, S. 599 f.; zu älterem Gebrauch mit in und con vgl. ebd., S. 486): 
(39) In questa gara si rivelano particolarmente bravi gli avversari, che, sebbene arrivando da risultati non esattamente incoraggianti, non si sentono sconfitti.

In diesem Spiel erweisen sich die Gäste als besonders erfolgreich, die, wörtl.: obwobl kommend von Ergebnissen nicht genau ermutigend (obwohl sie vorher nicht sehr ermutigende Ergebnisse erzielt haben), sich nicht als Verlierer füblen.

Eingeleitete Partizipialgruppen wie in (40) sind laut Serianni (1989, S. 609) zum Ausdruck temporaler Relationen geläufig. Allerdings scheinen hier mehr oder weniger lexikalisierte Redewendungen wie dopo mangiato einen großen Anteil zu haben:

(40) Dopo mangiato vado sempre a riposare. (aus Giacoma/Kolb 2009) wörtl.: Nach gegessen (nach dem Essen bzw. wenn ich gegessen habe) rube ich mich immer aus.

Ferner rechnet Serianni (1989, S. 609) auch Ausdrücke zu den Einleitungselementen, bei denen nicht klar ist, ob es sich um Subjunktoren oder um vorangestellte Adverbien handelt, wie appena ('gerade', 'kaum') in appena arrivato ('eben angekommen') oder una volta ('einmal', 'wenn', 'als') in una volta dato il consenso (wörtl.: 'einmal gegeben die Zustimmung').

Mit dem Verb arrivare habe ich einzelne Belege für eingeleitete Partizipialgruppen mit eigenem Subjekt gefunden:

(41) UDINE. La showgirl Sylvie Lubamba è accusata di truffa e indebito uso di carte di credito di proprietà di un ex parlamentare friulano eletto nel centro-destra. La soubrette 38enne avrebbe "sottratto" 20 mila euro dalla carta di credito dell'ex onorevole. La carta è stata poi bloccata quando arrivato il "conto".

UDINE. Das Showgirl Sylvie Lubamba ist wegen Betrugs und Missbrauchs der Kreditkearten eines ehemaligen Abgeordneten der Mitte-Rechts-Koalition aus Friaul angeklagt worden. Die 38-jährige Soubrette habe 20.000 Euro von der Kreditkarte des Ex-Abgeordneten ,abgezogen". Die Karte wurde später gesperrt, wörtl.: als angekommen die „Rechnung“ (als die Abrechnung gekommen war).

Solche Beispiele entsprechen aber nach dem übereinstimmenden Urteil meiner Informanten nicht den grammatischen Normen. Normgerecht ist demnach bei adverbialen Partizipialgruppen mit eigenem Subjekt ausschließlich die uneingeleitete Verwendung: arrivato il conto. Soll ein expliziter Konnektor verwendet werden, so ist ein finiter Nebensatz zu bilden: quando è arrivato il conto.

Ähnlich wie im Deutschen scheint im Italienischen, wenn die semantische Relation präzisiert werden soll, der finite Adverbialsatz das Aus- 
drucksmittel der Wahl zu sein. Adverbiale Gerundial- und Partizipialgruppen werden nur verwendet, wenn semantische Unterspezifiziertheit unproblematisch oder erwünscht ist und sich somit die Einleitung durch einen Konnektor erübrigt.

\subsection{Deutsch}

Im Deutschen bleibt man bei der semantischen Deutung der Relation zwischen einer uneingeleiteten adverbialen Partizipialgruppe und ihrem Matrixsatz stärker als in den romanischen Vergleichssprachen bei temporalsituierenden Lesarten (vgl. Zifonun/Hoffmann/Strecker 1997, S. 2219 f.):

(42) Die Genehmigung nach dem Bundeswasserstraßengesetz liegt erst seit dem 10. Januar 2008 vor. Dies vorausgeschickt, beantworte ich die Anfrage namens der Landesregierung wie folgt.

In (42) stellt die Partizipialgruppe den zeitlichen Kontext bereit, in dem der Sachverhalt stattfindet, der im Matrixsatz beschrieben wird: Der vorherige Satz wurde vorausgeschickt; nun wird die Anfrage beantwortet. Konditionale, kausale, konzessive und sonstige Fortinterpretationen solcher Ausdrücke sind zwar nicht ausgeschlossen, aber eher untypisch. Komplexere semantische Relationen zum Matrixsatz werden im Deutschen bevorzugt durch finite Nebensätze angezeigt. Ersatzweise können subjunktor-eingeleitete Partizipialgruppen in Frage kommen (vgl. Welker 1992, S. 64; Zifonun/Hoffmann/Strecker 1997, S. 2221; Gärtner 2003, S. 95):

(43) Alle Kurse finden, soweit nicht anders angegeben, in der Pädagogischen Akademie statt.

(44) Danke für den Hinweis, den ich selbst, da schlafend, nicht nutzen konnte.

In (43) wird durch den Subjunktor eine konditionale, in (44) eine kausale Lesart verlangt. Für temporal-situierende Relationen werden subjunktoreingeleitete Partizipialgruppen im Deutschen dagegen kaum verwendet:

(45) *Als fertig aufgebaut, wurde der Computer sofort in Betrieb genommen.

(46) ?Sobald angekommen, organisiert man sich am besten eine südafrikanische SIM-Karte. Damit kann man recht günstig telefonieren.

Formulierungen wie (46) findet man vereinzelt. Für mein Sprachgefühl sind sie nicht ungrammatisch, klingen aber gekünstelt und unidiomatisch. Deutsche Informanten, denen ich das Beispiel vorgelegt habe, haben diesen Eindruck durchweg bestätigt. 
In der syntaktischen Struktur subjunktor-eingeleiteter Partizipialgruppen besetzt der Subjunktor, wie in finiten Nebensätzen, die linke Klammerposition:

\begin{tabular}{|c|l|l|l|l|}
\hline VF & \multicolumn{1}{|c|}{ LK } & \multicolumn{1}{|c|}{ MF } & \multicolumn{1}{c|}{ RK } & NF \\
\hline & soweit & nicht anders & angegeben & \\
\hline & da & & schlafend & \\
\hline
\end{tabular}

Abb. 7: Linearstruktur subjunktor-eingeleiteter Partizipialgruppen im Deutschen

\section{Durch eine Präposition eingeleitete Infinitivgruppen}

\subsection{Portugiesisch}

Infinitive sind nominale Formen des Verbs (vgl. z.B. Bechara 1999, S. 224; Schwarze 1995, S. 206; Renzi et al. 2001, Bd. II, S. 559 ff.). In der traditionellen Grammatikschreibung werden erweiterte Infinitive häufig unmittelbar den Nebensätzen zugeschlagen und ihre Einleiter - etwa das deutsche um (vgl. Welker 1992, S. 252 f.; Duden 2009, S. 629 ff., 1092) oder das italienische per (vgl. Serianni 1989, S. 580) - entsprechend zu den Konjunktionen gerechnet (vgl. Bechara 1999, S. 518). In der jüngeren Grammatikschreibung des Portugiesischen ist man stärker auf die Unangemessenheit dieser Kategorisierung aufmerksam geworden und hat hervorgehoben, dass die Einleiter satzwertiger Infinitive als Präpositionen einzustufen sind (vgl. Bechara 1999, S. 471 f.; Mioto/Kato 2002, S. 394 ff.; Gärtner 2003, S. 96 ff.; GPB, Bd. II, 2008, S. 813; GPB, Bd. III, 2009, S. 206 ff.; dazu auch: Kortmann 1997, S. 66 f.).

Im Portugiesischen können adverbiale Infinitivgruppen mit einer groBen Zahl von (teils komplexen) Präpositionen eingeleitet werden, z.B. antes de ('vor'), depois de ('nach'), ao ('gleichzeitig mit') u.a. für temporale, para oder a fim de ('für', 'um') für finale, por ('wegen'), com ('mit') u.a. für kausale, apesar de ('trotz'), não obstante ('ungeachtet') u.a. für konzessive Verknüpfungen u.v.m. (vgl. Bechara 1999, S. 518 ff.; Mateus et al. 2003, S. 712 ff.). Ein Beispiel mit einer kausalen Infinitivgruppe:

(47) A psicoterapia ocupa hoje um lugar fundamental na área da saúde, por trazer uma visão integrada do homem, considerando as dimensões orgânicas, psíquicas e sociais conjuntamente.

Die Psychotherapie spielt heute eine zentrale Rolle auf dem Gebiet der Gesundbeit, wörtl.: für bringen eine Sicht ganzheitliche des Menschen (wegen ibres ganzheitlichen Menschenbildes), die/das organische, psychische und soziale Gesichtspunkte gleichermaßen berücksichtigt. 
Die Infinitivgruppe hat hier kein eigenes syntaktisches Subjekt. Das semantische Subjekt muss aus dem Kontext erschlossen werden. Infinitivgruppen können im Portugiesischen aber ebenso wie Partizipial- und Gerundialgruppen auch eigene syntaktische Subjekte haben. Wieder ein Beispiel aus einer Politikerbiographie:

(48) Seu corpo foi transladado para São Paulo e o trem especial no qual viajou parou a cada estação a fim de o ilustre político receber as últimas homenagens.

Sein Leichnam wurde nach São Paulo überführt, und der Sonderzug, in dem er reiste, bielt an jedem Bahnhof, wörtl.: zwecks der berühmte Politiker empfangen die letzten Ebrungen (damit der berühmte Politiker die letzten Ehrungen empfangen konnte).

Das Subjekt o ilustre político ('der berühmte Politiker') steht hier präverbal. Bei einem inakkusativen Verb müsste es postverbal stehen.

Eine vielbeachtete Besonderheit des Portugiesischen sind die flektierten Infinitivformen (vgl. Müller/Riemer 1998, S. 173 ff.; HundertmarkSantos Martins 1998, S. 171 ff.). Es handelt sich um Infinitive mit Personalendungen für die 2. Person Singular und für alle drei Personen im Plural (vgl. Bechara 1999, S. 224; Cunha/Cintra 2008, S. 408). Für Tempus sind diese Formen nicht markiert. Nach Bechara (1999, S. 286) werden flektierte Infinitive verwendet, wenn man Mehrdeutigkeiten in Bezug auf das semantische Subjekt des Infinitivs ausschließen will und wenn die Infinitivgruppe ein präverbales explizites Subjekt enthält (ebenso Cunha/Cintra 2008, S. 501 f. und detaillierter Hundertmark-Santos Martins 1998, S. 172 ff.). Insbesondere erleichtern es diese Formen, adverbiale Infinitivgruppen und Matrixsätze zu verknüpfen, die unterschiedliche Subjekte haben. Sie nähern Infinitivgruppen formal und semantisch sehr stark finiten Nebensätzen an (vgl. Gärtner 2003, S. 91). Ein Beispiel:

Para a polícia e os políticos terem alguma credibilidade eu sugiro que as drogas apreendidas sejam queimadas à vista da população. wörtl.: Für die Polizei und die Politiker haben-sie einige Glaubwürdigkeit (damit die Polizei und die Politiker ein Minimum an Glaubwürdigkeit behalten), schlage ich vor, dass konfiszierte Drogen vor den Augen der Bevölkerung verbrannt werden.

Hier ist a polícia e os políticos ('die Polizei und die Politiker') das Subjekt der Infinitivgruppe und $e \boldsymbol{u}$ ('ich') das Subjekt des Matrixsatzes.

Haben Infinitivgruppe und Matrixsatz das gleiche Subjekt, so kann der flektierte Infinitiv zur Verdeutlichung (vgl. Cunha/Cintra 2008, S. 503) dennoch gesetzt werden wie in (50b), in Kontrastkontexten auch mit explizitem Subjekt wie in (50a). Besteht kein solcher Bedarf, so wird im schriftsprachlichen Standard der kürzere nicht-flektierte Infinitiv wie in (50c) bevorzugt: 
(50a) Para nós sabermos como ler o texto, precisamos conhecer a sua tradução.

wörtl.: Für wir wissen-wir wie lesen den Text (damit wir wissen, wie wir den Text lesen sollen), müssen wir Ibre Übersetzung kennen.

(50b) Para sabermos como ler o texto, precisamos conhecer a sua tradução. wörtl.: Für wissen-wir wie lesen den Text ...

(50c) Para saber como ler o texto, precisamos conhecer a sua tradução. wörtl.: Für wissen wie lesen den Text ...

\subsection{Italienisch}

Im Italienischen gibt es keine flektierten Infinitivformen. Nicht-flektierte Infinitive können aber wie im Portugiesischen sehr frei durch Präpositionen eingeleitet und als Adverbialia verwendet werden. Renzi et al. (2001, Bd. II, S. 553 ff.) nennen die Präpositionen $a$, da, di, dopo ('nach'), fino a ('bis'), per ('wegen', 'um'), prima di ('vor'), senæa ('ohne') und tra ('zwischen') für temporale, konditionale, kausale, konsekutive, instrumentale, finale und konzessive Verknüpfungen. Hinzu kommen zahlreiche Wortgruppen mit präpositionaler Funktion wie in attesa di ('in Erwartung'), in maniera di ('um', 'damit'), invece di ('statt') u.v.m. Zwei Beispiele:

(51) Dopo avere visto i video, ti scriverò nuovamente.

wörtl.: Nach haben gesehen die Videos (wenn ich die Videos gesehen habe), schreibe ich dir wieder.

(52) Impareremo in questa guida a fare una catenella con la lana, in modo da cominciare a prendere confidenza con l'uncinetto.

In dieser Anleitung lernen wir, mit Wolle eine Luftmasche zu machen, wörtl.: in Weise zu beginnen zu fassen Vertranen mit dem Häkeln (um allmählich mit dem Häkeln vertraut zu werden).

In diesen Beispielen enthält die Infinitivgruppe kein eigenes Subjekt. Das semantische Subjekt des Infinitivs muss aus dem Kontext erschlossen werden. Präpositional eingeleitete Infinitivgruppen mit eigenem explizitem Subjekt sind im Italienischen selten und stilistisch markiert (vgl. Serianni 1989, S. 580, 608; Schwarze 1995, S. 206; Renzi et al. 2001, Bd. II, S. 484, 527 ff.). Nach Renzi et al. (ebd., S. 528) kann das Subjekt in solchen Fällen nicht unmittelbar vor dem Infinitiv stehen. In der Regel nimmt es die Position unmittelbar nach dem Infinitiv ein. Die Autoren geben (ebd., S. 555 f.) einen Beleg mit der Präposition per, den sie einem hohen, literarischen Stil zuordnen:

(53) L'amica, per essere Rosaria così lentigginosa, la chiamava semola di granturco. (E. Morante, Menzogna e sortilegio) 
Ihre Freundin, wörtl. für sein Rosaria so sommersprossig (da Rosaria so sommersprossig war), nannte sie Maisgrieß.

Nach Renzi et al. (2001, Bd. II, S. 528) können nur die Infinitive essere ('sein'), avere ('haben'), dovere ('müssen', 'sollen'), potere ('können', 'dürfen'), trattarsi ('sich handeln'), esistere ('existieren', 'bestehen') und spettare ('zukommen', 'zustehen') mit eigenem explizitem Subjekt auftreten. Einzelbelege aus dem Internet mit anderen Verben wurden von meinen Informanten als Substandard eingestuft:

(54) Purtroppo ormai penso che sei abituata a questi cambiamenti. Mi ricordo, quest'estate prima di arrivare tuo marito ci sono stati tanti ritardi.

Leider denke ich inzwischen, dass du dich an diese Veränderungen gewöhnt hast. Ich erinnere mich, dass es letzten Sommer, wörtl.: vor ankommen dein Mann (bevor dein Mann ankam), viele Verspätungen gab.

Renzi et al. (2001, Bd. II, S. 529) deuten an, dass explizite Subjekte bei Infinitiven für besondere informationsstrukturelle Zwecke dienen können. Hierzu scheint aber noch weitere Forschung notwendig zu sein.

\subsection{Deutsch}

Im Deutschen können adverbiale Infinitivgruppen nur mit um, obne und (an) statt eingeleitet werden (vgl. Zifonun/Hoffmann/Strecker 1997, S. 1430 ff.):

(55) Um Ihre Frau zu überzeugen, müssen Sie sich Mühe geben.

(56) Der Präsident hat das Angebot, ohne zu zögern, akzeptiert.

(57) Das Kind starb, weil die Eltern beteten, statt einen Arzt zu holen.

Der Infinitiv muss in solchen Konstruktionen stets mit der Partikel (bzw. dem Affix; vgl. Zifonun/Hoffmann/Strecker 1997, S. 2159; Sternefeld 2008, S. 89) zu stehen. Seit Bech (1983 [1955], S. 12) wird der zu-Infinitiv auch als Supinum im 2. Status bezeichnet.

Die Dudengrammatik (2009, S. 625, 629 ff., 853 f.) führt um, obne und (an) statt als Einleiter von Infinitivgruppen im Einklang mit der Tradition als Subjunktoren. Zifonun/Hoffmann/Strecker (1997, S. 1430) sprechen vorsichtiger von „Partikeln“. Von Subjunktoren möchte man erwarten, dass sie durch finite Nebensätze ergänzt werden können. Das trifft auf um, obne und (an) statt nicht zu:

(55a) *Um Sie Ihre Frau überzeugen, müssen Sie sich Mühe geben.

(56a) *Der Präsident hat das Angebot, ohne er zögerte, akzeptiert.

(57a) *Das Kind starb, weil die Eltern beteten, statt sie einen Arzt holten. 
Stattdessen können sie durch Nominalgruppen ergänzt werden, wie es für Präpositionen kennzeichnend ist:

(55b) Um die Zustimmung Ihrer Frau müssen Sie sich bemühen.

(56b) Der Präsident hat das Angebot ohne Verzögerung akzeptiert.

(57b) Das Kind starb, weil die Eltern einen Pfarrer holten statt eines Arztes.

Die Distribution spricht demnach dafür, Einleiter adverbialer Infinitivgruppen auch im Deutschen als Präpositionen einzustufen. Der Grund, warum dies in den meisten Grammatiken nicht geschehen ist, dürfte vor allem darin liegen, dass Infinitivgruppen im Deutschen eher untypische Realisierungsformen für Adverbialia sind. Dadurch ist es viel weniger auffällig, dass auch Präpositionen als Satzverknüpfer fungieren können (vgl. dazu Pasch et al. 2003, S. 4).

Stuft man um, obne und (an)statt gemäß der traditionellen Sicht als Subjunktoren ein, so müssten sie in der Linearstruktur der Infinitivgruppe die linke Klammerposition einnehmen. Allerdings können obne und (an)statt auch durch dass-Sätze ergänzt werden, in denen der Komplementierer dass die linke Klammerposition besetzt:

(56c) Der Präsident hat das Angebot, ohne dass er gezögert hätte, akzeptiert.

(57c) Das Kind starb, weil die Eltern beteten, statt dass sie einen Arzt holten.

In $(56 \mathrm{c} / 57 \mathrm{c})$ sollten obne und statt daher außerhalb des dass-Satzes stehen, der ihre Ergänzung bildet (vgl. Sternefeld 2008, S. 201 f.; für eine analoge Argumentation zum Portugiesischen vgl. Mioto/Kato 2002, S. 394 ff.):

\begin{tabular}{|l|l|l|l|l|l|}
\hline \multicolumn{1}{|c|}{ Präp } & VF & \multicolumn{1}{c|}{ LK } & \multicolumn{1}{c|}{ MF } & \multicolumn{1}{c|}{ RK } & \multicolumn{1}{c|}{ NF } \\
\hline ohne & & dass & er & gezögert hätte & \\
\hline statt & & dass & sie einen Arzt & holten & \\
\hline
\end{tabular}

Abb. 8: Linearstruktur präpositions-eingeleiteter dass-Sätze im Deutschen

Es ist naheliegend anzunehmen, dass Infinitivgruppen, die durch obne oder (an)statt eingeleitet werden, eine analoge Struktur aufweisen, dass also auch hier die Präposition ihren Sitz außerhalb der Infinitivgruppe hat und deren linke Klammerposition unbesetzt ist. Bei um, das nicht durch einen dass-Satz ergänzbar ist, ist die Datenlage weniger eindeutig. Da um sich aber nirgends wie ein Subjunktor verhält, ist auch hier meiner Meinung nach die Schlussfolgerung zwingend, dass es nicht in der linken Klammerposition, sondern nur außerhalb der Infinitivgruppe stehen kann: 


\begin{tabular}{|l|l|l|l|l|l|}
\hline \multicolumn{1}{|c|}{ Präp } & VF & LK & \multicolumn{1}{|c|}{ MF } & \multicolumn{1}{c|}{ RK } & NF \\
\hline ohne & & & & zu zögern & \\
\hline statt & & & einen Arzt & zu holen & \\
\hline um & & & Ihre Frau & zu überzeugen & \\
\hline
\end{tabular}

Abb. 9: Linearstruktur präpositions-eingeleiteter $2 u$-Infinitivgruppen im Deutschen

Ein eigenes formales Subjekt können Infinitivgruppen im Deutschen grundsätzlich nicht haben (vgl. Zifonun/Hoffmann/Strecker 1997, S. 2162 f.). Thr semantisches Subjekt muss aus dem Kontext erschlossen werden. Häufig bietet sich dafür das Subjekt des Matrixsatzes an wie in (58), aber (59) zeigt, dass es auch andere Möglichkeiten gibt. Hier ist als semantisches Subjekt ein generisches man zu interpolieren:

(58) Er beendete seine Mission und ging - nicht ohne die Alemannen verflucht zu haben - nach Italien. (gekürzt aus Zifonun/Hoffmann/ Strecker 1997, S. 1431)

(59) Anstatt das Geld in Säcken zu transportieren, sollte der Zahlungsverkehr besser bargeldlos erfolgen. (bearbeitet nach Zifonun/Hoffmann/Strecker 1997, S. 1433)

\section{Zusammenfassung und Ausblick}

Die Datenübersicht, die ich in diesem Aufsatz gegeben habe, führt zu drei Schlussfolgerungen:

(i) Verglichen mit dem Deutschen verfügen die romanischen Sprachen über eine größere Ausdrucksvielfalt bei Adverbialia mit verbalem Kern, das Portugiesische noch deutlicher als das Italienische.

(ii) Neben Subjunktoren spielen in den romanischen Sprachen Präpositionen eine wichtige Rolle als Satzverknüpfer.

(iii) Während im Deutschen eine relativ klare kategoriale Grenze zwischen satzförmigen und satzwertigen Ausdrücken gezogen werden kann, scheint in den romanischen Vergleichssprachen eher eine skalare Abstufung vorzuliegen.

\subsection{Ausdrucksvielfalt}

Die untersuchten Ausdrucksmittel - eingeleitete und uneingeleitete finite Nebensätze, eingeleitete und uneingeleitete Partizipial- und Gerundial- (bzw. Präsenspartizip-)gruppen, eingeleitete adverbiale Infinitivgruppen - stehen 
in allen drei Vergleichssprachen zur Verfügung. Es hat sich aber gezeigt bzw. bestätigt, denn die Beobachtung als solche ist nicht neu (vgl. z.B. Rudolph 1997, S. 331; Gärtner 2003, S. 92 ff.) - dass die romanischen Vergleichssprachen die Mittel gleichmäßiger und vielfältiger nutzen als das Deutsche. Im Portugiesischen ist die Ausdrucksvielfalt am größten.

Im Deutschen werden Adverbialia mit verbalem Kern bevorzugt als Nebensätze realisiert. Adverbiale Infinitivgruppen mit Präposition sind untypisch. Sie können nur mit drei Präpositionen gebildet und nur für wenige semantische Relationen genutzt werden. Adverbiale Partizipialgruppen werden als stilistisch knappere Alternative zu Nebensätzen oft zu Ökonomiezwecken eingesetzt: uneingeleitet für zeitlich-situierende, eingeleitet für komplexere Relationen.

Auch im Italienischen werden adverbiale Nebensätze für die ganze Bandbreite der Verknüpfungsrelationen verwendet. Daneben gibt es aber auch zahlreiche Präpositionen, mit denen adverbiale Infinitivgruppen gebildet werden können. Partizipial- und Gerundialgruppen werden überwiegend uneingeleitet verwendet und sind semantisch entsprechend unterspezifiziert. Eingeleitete Partizipial- und Gerundialgruppen sind untypisch.

Im Portugiesischen stehen adverbiale Nebensätze, Infinitivgruppen und Partizipial- bzw. Gerundialgruppen als gleichberechtigte Ausdrucksmittel nebeneinander. Mit Ausdrücken aller drei Klassen kann eine große Bandbreite semantischer Relationen abgedeckt werden. Neben unflektierten stehen auch flektierte Infinitivformen zur Verfügung, durch die die Infinitivgruppe größere semantische und informationsstrukturelle Eigenständigkeit gegenüber dem Matrixsatz erhält. Partizipial- und Gerundialgruppen können (zumindest im informellen brasilianischen Portugiesisch) auch eingeleitet verwendet werden, um die semantische Relation zum Matrixsatz stärker festzulegen.

Abbildung 10 gibt einen abschließenden Überblick über die behandelten Sprachmittel. Die Datenlage passt gut zu der Beobachtung Kortmanns (1997, S. 243), dass an den Rändern des west-/mitteleuropäischen Sprachraums stärker als im Zentrum Gebrauch von nicht-finiten Ausdrucksmitteln bei adverbialen Satzverknüpfungen gemacht wird. Kortmann bezieht sich dabei auf das Englische im Westen und das Russische im Osten. Das Portugiesische, das zur westlichen Peripherie des europäischen Sprachraums zu rechnen ist, ähnelt mit seiner Vielfalt an adverbialen Infinitiv-, Partizipial- und Gerundialgruppen dem Englischen. Das Deutsche, das finiten adverbialen Nebensätzen stets den Vorzug gibt, ist ein typischer Vertreter der mitteleuropäischen Sprachen. Das Italienische steht zwischen beiden, was bei den Verwendungsmöglichkeiten für eingeleitete Infinitiv- und uneingeleitete Partizipial- und Gerundialgruppen am deutlichsten wird. 


\begin{tabular}{|c|c|c|}
\hline $\begin{array}{l}\text { eingeleitete adv. } \\
\text { Nebensätze }\end{array}$ & $\begin{array}{l}\text { eingeleitete adv. } \\
\text { Infinitivgruppen }\end{array}$ & $\begin{array}{l}\text { eingeleitete adv. Partizipial- } \\
\text { bzw. Gerundialgruppen }\end{array}$ \\
\hline $\begin{array}{l}\text { dt. mit } 50-100 \text { Subjunktoren } \\
\text { volle Bandbreite sem. Rela- } \\
\text { tionen } \\
\text { stets subjekthaltig }\end{array}$ & $\begin{array}{l}\text { dt. nur mit } 3 \text { Präpositionen } \\
\text { nur wenige sem. Relationen } \\
\text { stets subjektlos }\end{array}$ & $\begin{array}{l}\text { dt. mit verschiedenen Subjunk- } \\
\text { toren } \\
\text { konditional, kausal, konzes- } \\
\text { siv u.a. } \\
\text { stets subjektlos }\end{array}$ \\
\hline $\begin{array}{l}\text { it. mit } 50-100 \text { Subjunktoren } \\
\text { volle Bandbreite sem. Rela- } \\
\text { tionen } \\
\text { stets subjekthaltig }\end{array}$ & $\begin{array}{l}\text { it. mit zahlreichen Präpositio- } \\
\text { nen } \\
\text { zahlreiche sem. Relationen } \\
\text { in der Regel subjektlos }\end{array}$ & it. untypisch \\
\hline $\begin{array}{l}\text { pt. mit } 50-100 \text { Subjunktoren } \\
\text { volle Bandbreite sem. Rela- } \\
\text { tionen } \\
\text { stets subjekthaltig }\end{array}$ & $\begin{array}{l}\text { pt. mit zahlreichen Präpositio- } \\
\text { nen } \\
\text { zahlreiche sem. Relationen } \\
\text { subjektlos oder subjekthaltig } \\
\text { Subjekt präverbal oder post- } \\
\text { verbal } \\
\text { nicht-flektierte u. flektierte } \\
\text { Infinitive }\end{array}$ & $\begin{array}{l}\text { pt. informell } \\
\text { mit verschiedenen Subjunk- } \\
\text { toren } \\
\text { verschiedene sem. Relatio- } \\
\text { nen } \\
\text { meist subjektlos }\end{array}$ \\
\hline $\begin{array}{l}\text { uneingeleitete adv. } \\
\text { Nebensätze }\end{array}$ & & $\begin{array}{l}\text { uneingeleitete adv. Partizi- } \\
\text { pial- bzw. Gerundialgruppen }\end{array}$ \\
\hline $\begin{array}{l}\text { dt. unterschiedl. sem. Relationen } \\
\text { stets subjekthaltig }\end{array}$ & & $\begin{array}{l}\text { dt. nur zeitlich-situierend } \\
\text { stets subjektlos }\end{array}$ \\
\hline $\begin{array}{l}\text { it. } \\
\text { nulten } \\
\text { nur konditional-kontrafak- } \\
\text { tisch } \\
\text { stets subjekthaltig }\end{array}$ & & $\begin{array}{l}\text { it. zeitlich, konditional, kausal } \\
\text { u.a. } \\
\text { subjektlos oder subjekthaltig } \\
\text { Subjekt in der Regel post- } \\
\text { verbal }\end{array}$ \\
\hline $\begin{array}{l}\text { pt. nur konditional-kontrafak- } \\
\text { tisch } \\
\text { stets subjekthaltig }\end{array}$ & & $\begin{array}{l}\text { pt. zahlreiche sem. Relationen } \\
\text { subjektlos oder subjekthaltig } \\
\text { Partizipien: Subjekt i.d.R. } \\
\text { postverbal } \\
\text { Gerundien: Subj. prä- o. } \\
\text { postverbal }\end{array}$ \\
\hline
\end{tabular}

Abb. 10: Synopse der Ausdrucksmittel für Adverbialia mit verbalem Kern im Deutschen, Italienischen und Portugiesischen

\subsection{Subjunktoren und Präpositionen}

Präpositionen, die typischerweise durch Nominalgruppen ergänzt werden, werden in der deutschen Grammatikschreibung traditionell nicht als Satzverknüpfer behandelt (vgl. Pasch et al. 2003, S. 4). Subjunktoren und Konjunktoren, die Sätze einleiten können, werden als Konjunktionen zusammengefasst. Dass Subjunktoren, bei Licht betrachtet, mehr syntaktische und 
semantische Eigenschaften mit den Präpositionen als mit den Konjunktoren teilen (vgl. Jespersen 1924, S. 89; Wiese 1978, S. 144 f.), wird durch diese Tradition verdeckt.

Anhänger der traditionellen Wortarteinteilung können sich zu ihrer Rechtfertigung darauf berufen, dass Präpositionen im Deutschen empirisch kaum eine Rolle als Satzverknüpfer spielen. Die wenigen Ausdrücke, die sowohl durch Nominalgruppen als auch durch finite Nebensätze ergänzbar sind (während, seit und bis), werden im einen Fall als Präpositionen, im andern als Subjunktoren kategorisiert. Die wenigen Ausdrücke, die durch Nominalgruppen oder durch satzwertige Infinitive ergänzt werden können (um, obne und (an)statt), werden einfachheitshalber nach dem gleichen Muster behandelt.

Der Blick auf Vergleichssprachen wie Italienisch und Portugiesisch, in denen Subjunktoren und Präpositionen systematisch parallele Inventare bilden, wobei erstere durch finite und letztere durch infinite Ausdrücke zu ergänzen sind, kann dazu anregen, auch für das Deutsche die (bescheidene) Rolle von Präpositionen als Satzverknüpfer anzuerkennen. Dann können um, ohne und (an) statt ohne weiteres zugleich das eine und das andere sein. ${ }^{10}$

\subsection{Satzförmige und satzwertige Ausdrücke}

Wenn man nur das Deutsche betrachtet, scheint zwischen satzförmigen und satzwertigen Ausdrücken eine relativ klare kategoriale Grenze zu liegen: Satzförmige Ausdrücke haben eine finite Verbform als Kern und enthalten ein eigenes explizites Subjekt; satzwertige Ausdrücke haben eine infinite Verbform als Kern und sind formal subjektlos.

Der Vergleich mit den romanischen Sprachen wirft auch auf diese Abgrenzung ein relativierendes Licht. Zum einen können sowohl im Italienischen als auch im Portugiesischen Partizipial- und Gerundialgruppen problemlos eigene Subjekte haben. Zum anderen können im Portugiesischen (weniger deutlich im Italienischen) auch Infinitivgruppen eigene Subjekte haben. Drittens können im Portugiesischen Infinitivformen für Person und Numerus flektiert werden. Übereinzelsprachlich gesehen, scheint zwischen satzförmigen und satzwertigen Ausdrücken also eher ein Kontinuum als eine scharfe Grenze zu liegen, ähnlich wie es von verschiedenen Autoren für die Unterscheidung zwischen Koordination und Subordination postuliert wurde (vgl. Kortmann 1997, S. 56 ff.; Wegener 2001).

10 In romanischen Grammatiken des Deutschen findet man um, obne und (an)statt mit zu-Infinitiv in der Tat öfters als Präpositionen eingeordnet, etwa in der französischen Grammatik von Schanen/Confais (1989, S. 280). 


\section{Literatur}

Axel, Katrin/Wöllstein, Angelika (2009): German verb-first conditionals as unintegrated clauses: A case study in converging synchronic and diachronic evidence. In: Winkler, Susanne/Featherston, Sam (Hg.): The Fruits of Empirical Linguistics. Bd. 2: Product. (= Studies in Generative Grammar 102). Berlin u.a., S. 1-35.

Bech, Gunnar (1983 [1955]): Studien über das deutsche Verbum infinitum. 2., unveränd. Aufl. (mit einem Vorwort von Cathrine Fabricius-Hansen). (= Linguistische Arbeiten 139). Tübingen. [1. Aufl. 1955 Kopenhagen (= Historisk-filologiske meddelelser 35,2)].

Bechara, Evanildo (1999): Moderna Gramática Portuguesa. 37., überarb. u. erg. Aufl. Rio de Janeiro.

Blühdorn, Hardarik (2010): A semantic typology of sentence connectives. In: Harden, Theo/Hentschel, Elke (Hg.): 40 Jahre Partikelforschung. (= Stauffenburg Linguistik 55). Tübingen, S. 215-231.

Braga, Maria Luiza (1996): Processos de redução: o caso das orações de gerúndio. In: Koch, Ingedore G. Villaça (Hg.): Gramática do Português Falado. Bd. VI: Desenvolvimentos. Campinas, S. 231-251.

Cunha, Celso/Cintra, Lindley (2008): Nova Gramática do Português Contemporâneo. 5. Aufl. Rio de Janeiro.

Duden (2009): Duden. Bd. 4: Die Grammatik. 8., überarb. Aufl. Hrsg. v.d. Dudenredaktion. Mannheim u.a.

Endruschat, Annette (2004): „Lavou-se, enfiou a roupa de casa, veio para a biblioteca, sentou-se a uma cadeira de balanço, descansando": zur Übersetzung portugiesischer Gerundialkonstruktionen in verschiedenen Textsorten. In: Endruschat, Annette/Schönberger, Axel (Hg.): Übersetzung und Übersetzen aus dem und ins Portugiesische. Akten des 4. Deutschen Lusitanistentags 2001, 11.-14.9.2001, Germersheim. (= Beihefte zu Lusorama: Reihe 1, Studien zur portugiesischen Sprachwissenschaft 10). Frankfurt a.M., S. 269-303.

Gärtner, Eberhard (1998): Grammatik der portugiesischen Sprache. Tübingen.

Gärtner, Eberhard (2003): Konjunktion = Konjunktion, Präposition = Präposition? Eine vergleichende Untersuchung zu Funktionswörtern im Deutschen und Portugiesischen. In: Blühdorn, Hardarik/Schmidt-Radefeldt, Jürgen (Hg.): Die kleineren Wortarten im Sprachvergleich Deutsch-Portugiesisch. (= Rostocker romanistische Arbeiten 7). Frankfurt a.M., S. 89-113.

Giacoma, Luisa/Kolb, Susanne (2009): Il Nuovo Dizionario di Tedesco. Dizionario Tedesco-Italiano, Italienisch-Deutsch. CD-ROM. 2. Aufl., Bologna/Stuttgart.

GPB II/III = Gramática do Português Culto Falado no Brasil. Gesamtleitung: Ataliba T. de Castilho. Bd. II: Classes de Palavras e Processos de Construção (2008). Hrsg. v. Ilari, Rodolfo/Neves, Maria Helena M. Bd. III: A Construção da Sentença (2009). Hrsg. v. Kato, Mary A./do Nascimento, Milton. Campinas. 
Höhle, Tilman N. (1986): Der Begriff „Mittelfeld“. Anmerkungen über die Theorie der topologischen Felder. In: Schöne, Albrecht (Hg.): Kontroversen, alte und neue. Akten des VII. Internationalen Germanisten-Kongresses Göttingen 1985. Bd. 3: Textlinguistik contra Stilistik?/Wortschatz und Wörterbuch/Grammatische oder pragmatische Organisation von Rede? Hrsg. v. Walter Weiss, Herbert Ernst Wiegand, Marga Reis. Tübingen, S. 329-340.

Holler, Anke (2007): Uniform oder different? Zum syntaktischen Status nicht-restriktiver Relativsätze. In: Deutsche Sprache 35, S. 250-270.

Hundertmark-Santos Martins, Maria Teresa (1998): Portugiesische Grammatik. 2., verb. Aufl. Tübingen.

Jespersen, Otto (1924): Philosophy of Grammar. London.

Kortmann, Bernd (1997): Adverbial Subordination. A typology and history of adverbial subordinators based on European languages. (= Empirical Approaches to Language Typology 18). Berlin u.a.

Mateus, Maria Helena Mira/Brito, Ana Maria/Duarte, Inês/Faria, Isabel Hub et al. (2003): Gramática da Língua Portuguesa. 6. Aufl., Lisboa.

Mioto, Carlos/Kato, Mary (2002): Aspectos sintáticos da subordinação sentencial. In: Abaurre, Maria Bernadete M./Rodrigues, Angela C.S. (Hg.): Gramática do Português Falado. Bd. VIII: Novos estudos descritivos. Campinas, S. 379-411.

Müller, Natascha/Riemer, Beate (1998): Generative Syntax der romanischen Sprachen. Französisch, Italienisch, Portugiesisch, Spanisch. Tübingen.

Neves, Maria Helena de Moura (2000): Gramática de Usos do Português. São Paulo.

Pasch, Renate/Brauße, Ursula/Breindl, Eva/Waßner, Ulrich Hermann (2003): Handbuch der deutschen Konnektoren. Linguistische Grundlagen der Beschreibung und syntaktische Merkmale der deutschen Satzverknüpfer (Konjunktionen, Satzadverbien und Partikeln). (= Schriften des Instituts für Deutsche Sprache 9). Berlin/New York.

Ravetto, Miriam/Blühdorn, Hardarik (2011): Die Kausalkonjunktionen denn, weil, da im Deutschen und poiché, perché, siccome im Italienischen. In: Ferraresi, Gisella (Hg.): Konnektoren im Deutschen und im Sprachvergleich. Beschreibung und grammatische Analyse. (= Studien zur Deutschen Sprache 53). Tübingen, S. 207-250.

Reis, Marga/Wöllstein, Angelika (2010): Zur Grammatik (vor allem) konditionaler V1Gefüge im Deutschen. In: Zeitschrift für Sprachwissenschaft 29, S. 111-179.

Renzi, Lorenzo/Salvi, Giampaolo/Cardinaletti, Anna et al. (2001): Grande Grammatica Italiana di Consultazione. Neuauflage. Bd. I-III. Bologna.

Rudolph, Elisabeth (1997): Gemeinsamkeiten und Unterschiede im literarischen Gebrauch von Satzverknüpfungen im Spanischen, Portugiesischen und Deutschen. In: Lüdtke, Helmut/Schmidt-Radefeldt, Jürgen (Hg.): Linguistica contrastiva. Deutsch versus Portugiesisch - Spanisch - Französisch. (= Acta romanica 9). Tübingen, S. 325-348.

Schanen, François/Confais, Jean-Paul (1989): Grammaire de l'allemand. Formes et fonctions. Paris. 
Schwarze, Christoph (1995): Grammatik der italienischen Sprache. 2., verb. Aufl., Tübingen.

Serianni, Luca (1989): Grammatica Italiana. Italiano comune e lingua letteraria. Torino.

Simões, José da Silva (2009): A Gramaticalização das orações de gerúndio no Português Brasileiro do séc. XVIII ao séc. XX. In: Castilho, Ataliba Teixeira de (Hg.): História do Português Paulista. Série Estudos Bd. I. Campinas, S. 417-463.

Soffritti, Marcello (2010): Der gerundio im italienischen Codice Civile und seine Entsprechungen in der Südtiroler Übersetzung. In: Foschi Albert, Marina et al. (Hg.): Text und Stil im Kulturvergleich. Pisaner Fachtagung 2009 zu interkulturellen Wegen Germanistischer Kooperation. München, S. 248-280.

Sternefeld, Wolfgang (2008): Syntax. Eine morphologisch motivierte generative Beschreibung des Deutschen. 2 Bde. 3., überarb. Aufl. (= Stauffenburg Linguistik 31). Tübingen.

Wegener, Heide (2001): Integration und Nichtintegration von Satzkonjunkten im Deutschen und Französischen. In: Haßler, Gerda (Hg.): Sprachkontakt und Sprachvergleich. (= Studium Sprachwissenschaft 34). Münster, S. 89-105.

Welker, Herbert Andreas (1992): Gramática Alemã. Brasília.

Wiese, Bernd (1978): Die Koordinationsrelation im Deutschen. Vorarbeiten zu ihrer Bestimmung. Magisterarbeit. Berlin, Freie Universität.

Zifonun, Gisela/Hoffmann, Ludger/Strecker, Bruno et al. (1997): Grammatik der deutschen Sprache. 3 Bde. (= Schriften des Instituts für Deutsche Sprache 7). Berlin. 
